\title{
Supernova detection and real-time alerts with the KM3NeT neutrino telescopes
}

\author{
Massimiliano Lincetto* \\ Aix Marseille Univ, CNRS/IN2P3 CPPM, Marseille (France) \\ E-mail: lincetto@cppm.in2p3.fr
}

Marta Colomer Molla

APC, Univ Paris Diderot, CNRS/IN2P3, CEA/Irfu, Obs de Paris, Sorbonne Paris Cité, France

IFIC - Instituto de Física Corpuscular (CSIC-Universitat de València), Paterna, Valencia,

E-mail: mcolomereapc.in2p3.fr

\section{on behalf of the KM3NeT Collaboration.}

The detection of a neutrino burst from the next Galactic Core-Collapse SuperNova (CCSN) will yield major breakthroughs in different fields of fundamental physics. The KM3NeT ORCA and ARCA neutrino telescopes in the Mediterranean Sea are expected to observe a significant number of neutrino interactions through the detection of Cherenkov light mostly induced by inverse beta decay processes in sea water. The detection of photons in coincidence between the 31 photomultipliers of KM3NeT digital optical modules (DOMs) allows to discriminate the CCSN signal against radioactive decays, bioluminescence and atmospheric muon backgrounds.

The detection sensitivity and the potential to resolve the time profile of the neutrinos have been studied by means of accurate Monte Carlo simulations coupled to in-depth studies of the KM3NeT background features. Online triggering criteria have been determined to send real-time alerts for low-energy CCSN neutrino bursts, and also to meet the requirements for the integration in the SNEWS global alert network. In view of future developments, a preliminary study has been conducted on the determination of the neutrino arrival time and the fast triangulation of the source by different detectors to constrain the CCSN localisation in the sky.

The New Era of Multi-Messenger Astrophysics - Asterics2019

25 - 29 March, 2019

Groningen, The Netherlands

\footnotetext{
* Speaker.
} 


\section{The KM3NeT neutrino telescopes}

The KM3NeT ARCA and ORCA Cherenkov neutrino telescopes are under construction in two underwater sites in the Mediterranean Sea [1]. The core element of KM3NeT detectors are the digital optical modules (DOM, [2]) featuring 31 directional photomultiplier tubes (PMTs) distributed throughout the surface of a spherical glass sphere. DOMs are connected in groups of 18 to form a vertical detection unit (DU) and a group of 115 DUs forms a building block. ORCA, off the French coast, will consist of one building block with a denser granularity and is primarily aimed at the determination of the neutrino mass ordering with a sensitivity on the tens-of-GeV energy range. ARCA, on the Italian site, will be composed by two building blocks with larger spacing between DOMs, and is aimed to the search for cosmic neutrino sources in the TeV-PeV energy range. Together, both detectors will comprise an instrumented volume reaching the $\mathrm{km}^{3}$ scale, providing at any time the full Southern hemisphere coverage over a wide energy range. The submarine infrastructure is ready at both sites, each currently operating one DU. New deployments are planned in the course of the year.

\section{Detector response to CCSN neutrinos}

Neutrino fluxes from state-of-the-art CCSN 3D simulations by the MPA Garching Group [3] are used to calculate the time and energy dependent neutrino interaction rates in sea water. The relevant interaction channels in water are inverse beta decay $(97 \%)$ followed by elastic scattering on electrons $(3 \%)$ and interactions with oxygen nuclei $(<1 \%)$ [4]. The response of the detector to neutrino interactions is simulated with GEANT4 and custom KM3NeT software.

CCSN neutrino interactions result in higher counting rates of individual PMTs as well as in the detection of photons in coincidence between different PMTs of the same DOM. In fact, photons from Cherenkov light emissions impinge on the PMTs almost simultaneously, resulting in nanosecond-scale coincidences that allow for an effective dis-

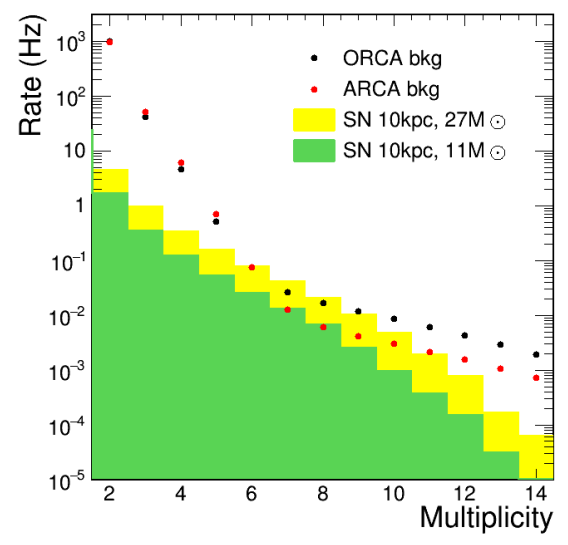

Figure 1: Single DOM coincidence rates as a function of multiplicity for background and CCSN signal in ARCA and ORCA for a 14 and a $27 \mathrm{M}_{\odot}$ progenitor. Muon-induced coincidence rates are lower in ARCA due to the larger depth of the site. tinction from the uncorrelated bioluminescence background. The number of PMT detecting photons in coincidence is called multiplicity (M), and can be used to identify the different kinds of Cherenkov emissions depending on their topology and light yield. The typical coincidence rates as a function of $\mathrm{M}$ are shown in Figure 1. Radioactive decays, mostly from ${ }^{40} \mathrm{~K}$, in sea water dominate on $M \leq 5$ and are used for in-situ calibration, while atmospheric muons are dominant in the $M \geq 8$ region. Simulations allow us 
to determine the best coincidence selection for the discrimination of the CCSN neutrino signal, resulting in the multiplicity interval $6 \leq M \leq 10$.

\section{Background reduction and sensitivity estimation}

In the given multiplicity region, downgoing atmospheric muons are still a major source of background. The detection of $M \geq 4$ coincidences on multiple DOMs correlated on a microsecond time scale is a clear signature of a muon-induced Cherenkov emission. This approach works especially well with the ORCA geometry, where the background is reduced almost to the residual

${ }^{40} \mathrm{~K}$ contribution, having a signature indistinguishable from the signal. Further optimisations are foreseen for ARCA.

After the rejection of the muon-like events, the trigger level for the CCSN detection is defined as the number of coincidences, in the given multiplicity selection, over a time window of duration $\tau$ (typically $\sim 500 \mathrm{~ms}$ ) following the onset of the event. Given the background rate $\rho_{B}$, estimated from the first ARCA and ORCA data, the significance of an observation $X=X_{d}$ is estimated from the p-value:

$$
P\left(X \geq X_{d}\right)=\sum_{X=X_{d}}^{+\infty} \mathscr{P}\left(\rho_{B} \cdot \tau, X\right)
$$

with $\mathscr{P}$ being the Poisson distribution. $X_{d} \propto d^{-2}$ is in this case the signal expectation for a CCSN neutrino burst from a progenitor at a distance $d$. The estimated sensitivity is shown in Figure 2: $5 \sigma$ sensitivity is reached beyond the Galactic Centre for a $11 \mathrm{M}_{\odot}$ progenitor and at $25 \mathrm{kpc}$ (full Galaxy coverage) for a $27 \mathrm{M}_{\odot}$ progenitor.

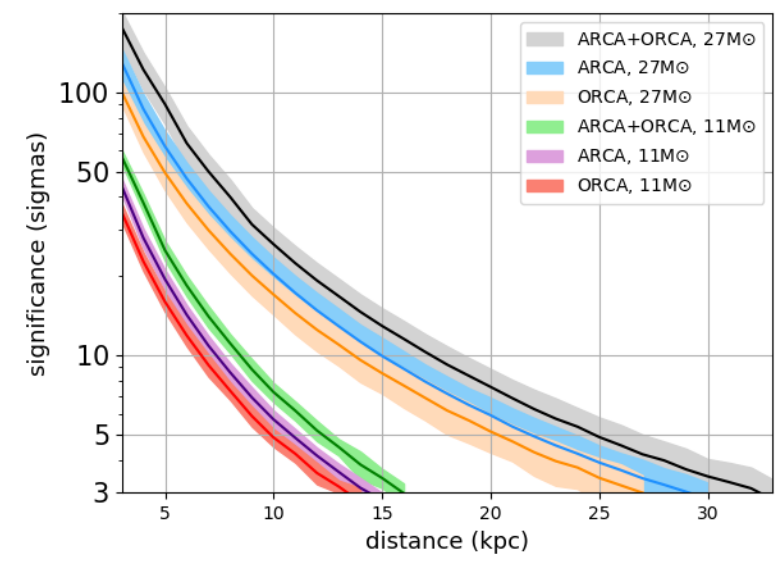

Figure 2: Detection significance for a CCSN neutrino signal from two progenitor assumptions as a function of the distance of the source for ORCA, ARCA, and the combination of the two detectors.

\section{Real-time transient search and SN alerts}

The online detection is based on the continuous evaluation of the trigger level over a sliding time window of width $\tau=400 \mathrm{~ms}$ updated with a frequency of $f_{s}=10 \mathrm{~Hz}$. With this approach, the 
p-value in equation 3.1 is converted into a false trigger rate $R_{B}$ dependent on the signal expectation $X_{d}$ :

$$
R_{B}\left(X \geq X_{d}\right)=f_{s} \cdot P\left(X \geq X_{d}\right)
$$

A $10 \mathrm{~Hz} f_{s}$ is a good compromise between the signal loss produced by the time discretisation and the resulting trial factor in the time domain. Additional robustness is ensured by counting up to one event per DOM in the given time window. The false alert rate as a function of $d$ is shown in Figure 3, where the maximum rate to participate in the global SNEWS alert network [5] (one in two weeks) is indicated. The full analysis of the first KM3NeT ORCA and ARCA data has shown that the background distribution perfectly follows the model prediction.

The all-data-to-shore DAQ concept used by KM3NeT provides a high customisability for the online and offline data analyses. A data stream dedicated to the supernova detection contains all the coincidences with $M \geq 4$ and represents the only coincidence data continuously recorded for permanent storage. The stream is processed in real time on each on-shore computing farm, producing summary information that is monitored by operators and relayed to a common endpoint where the real-time combined trigger level (ARCA and ORCA) is being tested. With the current detector configuration, a CCSN occurring within $4 \mathrm{kpc}$ would be observed with $a \geq 5 \sigma$ significance.

A buffering system for low-level data is under development in order to permanently store a snapshot of all coincidences around the time of the burst in case of an external alert or a self-generated trigger. Among the current analyses being developed, low-

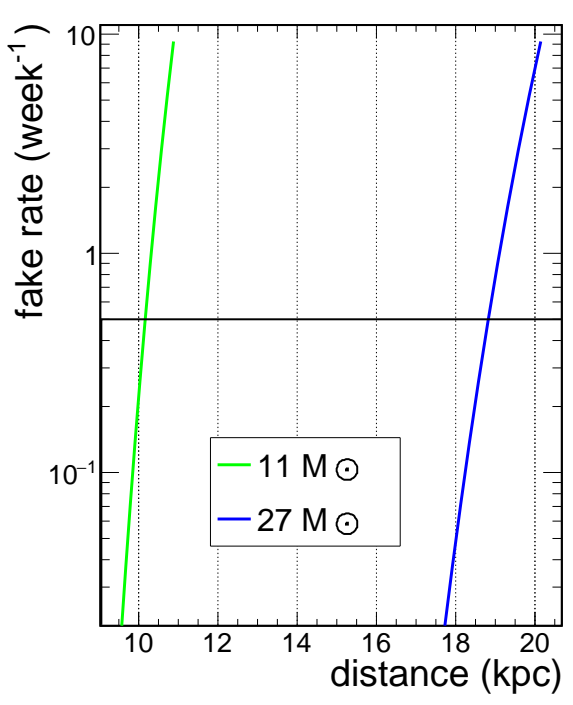

Figure 3: Estimated false trigger rates in ORCA for a background level equal or greater to the expectation value of signal plus background as a function of the source distance for the two progenitors. The horizontal black line indicates the SNEWS threshold. multiplicity coincidences are used in the study of fast time variations in the neutrino light curve [6] and the determination of the time of arrival of the burst, which is a crucial input for a precise localization of the CCSN on the sky and for a joint search for gravitational-wave signal [7].

The general architecture of the real-time system is outlined in Figure 4. A common infrastructure is shared between the supernova trigger and the online neutrino search. The design concept is based on a common dispatcher used by multiple clients to receive and send data, making the outputs of all the processing stages simultaneously available to any client. This structure allows for the building of flexible and extensible data processing pipelines. 


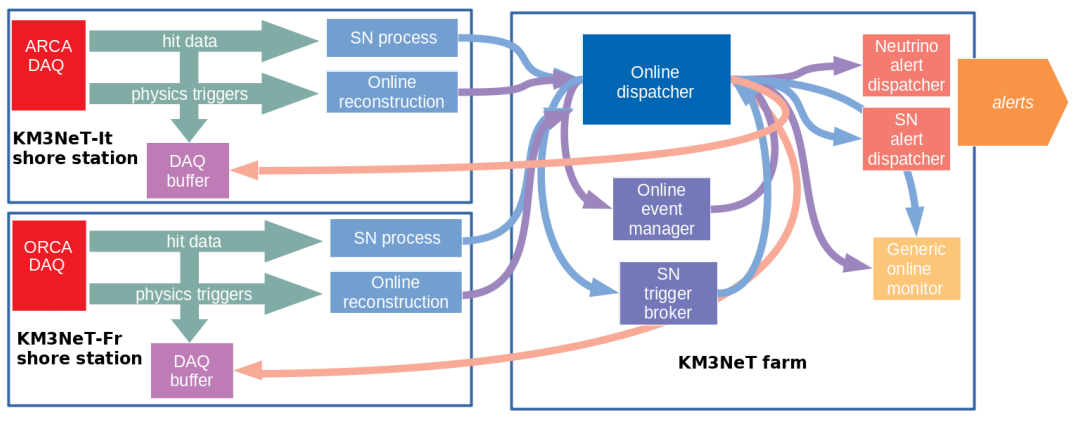

Figure 4: Functional diagram of the KM3NeT DAQ system and online framework outlining the information exchange between the two shore stations and the central farm dedicated to the realtime processing applications.

\section{Conclusion}

The sensitivity of the KM3NeT detectors to CCSN neutrino bursts has been evaluated to be of $5 \sigma$ at $25 \mathrm{kpc}$ (covering the full Galaxy) for a $27 \mathrm{M}_{\odot}$ stellar progenitor for the combination of the ORCA and ARCA detector, and beyond the Galactic Center in case of a $11 \mathrm{M}_{\odot}$ stellar progenitor and a single building block. A detection algorithm has been implemented as a real-time trigger that will allow to fulfill the SNEWS requirement for the false alert rate covering up to the Galactic Centre for the $11 \mathrm{M}_{\odot}$ progenitor and up to $18 \mathrm{kpc}$ for the $27 \mathrm{M}_{\odot}$ progenitor when considering a single ORCA-like building block. The prototype monitoring system is operational at the ORCA and ARCA shore stations, with one detection unit active at each site, and the commissioning of the online framework is in progress, where the real-time combination of the two detectors is envisaged. The study of the sensitivity to fast time variations in the neutrino light curve and to the determination of the time of arrival of the burst is being developed as the next step of the analysis.

\section{Acknowledgements}

This work was partly supported through the KM3NeT-INFRADEV (grant 739560) and ASTERICS (grant 653477) projects funded by the European Commission under the H2020 programme, and the LabEx UnivEarthS exploratory project E9: Low energy astrophysics with KM3NeT.

\section{References}

[1] KM3NeT Collaboration, Journal of Physics G 43 (8), 084001 (2016)

[2] KM3NeT Collaboration, Eur. Phys. J. C (2016) 76:54 (2015)

[3] I. Tamborra et al., Phys. Rev. D 90, 045032 (arXiv e-prints 1406.0006) (2014)

[4] K. Scholberg, Ann. Rev. Nucl. Part. Sci. 62 (2012) 81 doi:10.1146/annurev-nucl-102711-095006

[5] K. Scholberg, Astron. Nachr. 329 (2008) 337 doi:10.1002/asna.200710934

[6] A. Marek, H.-T. Janka and E. Müller, Astron. Astrophys. 496, 66 (2009)

[7] Nakamura et al., MNRAS 461, (2016) 